\title{
Predicting Trait Emotional Intelligence from HEXACO Personality: Domains, Facets, and The General Factor of Personality Jeromy Anglim ${ }^{1,}$ Gavin Morse ${ }^{1}$, Patrick D. Dunlop ${ }^{2}$, Amirali Minbashian ${ }^{3}$, Andrew Marty ${ }^{4}$
}

\section{Abstract *}

Objective: The current study provides a comprehensive analysis of the overlap between trait emotional intelligence (EI) and personality. This overlap was examined using the HEXACO personality framework at both the domain and facet levels, and through varying methods of deriving a General Factor of Personality (GFP). Method: A sample of 1370 Australian adults (51\% male, age in years $M=45.5, S D$ $=11.7$, range: 21 to 71 ) completed the 200-item HEXACO Personality Inventory-Revised and the Modified Assessing Emotions Scale measure of selfreported Trait EI. Results: The strongest domain correlations with Total EI emerged for extraversion $(r=$ .67) followed by openness $(r=.39)$, conscientiousness $(r$ $=.35)$, and agreeableness $(r=.26)$. Large adjusted

* Citation Information: Check for updates regarding year, issue and page numbers. Anglim, J., Morse, G., Dunlop, P. D., Minbashian, A., \& Marty, A. (2019). Predicting trait emotional intelligence from HEXACO personality: Domains, facets, and the general factor of personality. Journal of Personality. https://doi.org/10.1111/jopy.12493

1 School of Psychology, Deakin University, Geelong, Australia.

${ }^{2}$ Future of Work Institute, Faculty of Business and Law, Curtin University, Kent Street, Bentley, Western Australia, Australia, 6102

3 UNSW Business School, UNSW Sydney, Sydney, New South Wales, 2052, Australia

4 SACS Consulting, 350 Collins Street, Melbourne, Victoria, Australia, 3000

Author Note. Data, data analysis scripts, supplementary materials, and item-level information is provided at https://osf.io/uwdgs/ Correspondence concerning this article should be addressed to Jeromy Anglim, School of Psychology, Deakin University, Locked Bag 20000, Geelong, 3220, Australia. Email:

jeromy.anglim@deakin.edu.au 
multiple correlations were obtained when predicting Total EI from HEXACO domains (.74) and facets (.81). The correlations of the GFP and Total EI ranged from .53 to .64 depending on how the GFP was operationalized. Conclusion: Trait EI is largely captured by the HEXACO personality framework, whereby extraversion or the GFP provides a rough initial approximation, but composites of domains and facets provide progressively better representations.

Keywords: trait emotional intelligence, HEXACO, personality, general factor of personality, narrow traits

\section{Introduction}

Trait Emotional Intelligence (EI) has been defined as a "constellation of emotion-related selfperceptions and dispositions located at the lower levels of personality hierarchies" (Petrides, Pérez-González, \& Furnham, 2007, p. 26). Researchers have long been interested in the relationship between Trait EI and traditional personality frameworks (Joseph \& Newman, 2010; Petrides \& Furnham, 2001; van der Linden et al., 2017). Understanding this relationship helps to mutually define the two constructs. More critically, the degree of overlap between personality and Trait EI speaks to whether the construct of Trait EI offers anything unique (Zeidner, Roberts, \& Matthews, 2008). This is important both for researchers seeking to understand individual differences in human behavior and practitioners seeking to efficiently measure characteristics for such purposes as counselling, professional development, and highstakes selection.

While the majority of research examining the relationship between Trait EI and personality has focused on the broad dimensions of the Big 5 (see the metaanalysis by Joseph \& Newman, 2010), there has been resurgent interest in the overlap with alternative representations and levels of the personality hierarchy. These include narrow personality traits, alternatives to the Big 5 such as the HEXACO model (Ashton, Lee, \& De Vries, 2014), and higher-order personality traits such as the General Factor of Personality (GFP). Individual studies have obtained observed correlations between the GFP and Trait EI ranging from .65 to .78 (PérezGonzález \& Sanchez-Ruiz, 2014; Van der Linden, Tsaousis, \& Petrides, 2012). More recently, van der Linden et al. (2017) conducted a meta-analysis of 
correlations between the Big 5 and Trait EI and estimated a latent correlation between the GFP and Trait EI of around .85 . They concluded that both constructs measure social effectiveness and that "the GFP is very similar, perhaps even synonymous, to Trait EI" (p. 36). Alternatively, two recent studies (Austin \& Vahle, 2016; Veselka et al., 2010) correlating the six domains of HEXACO personality with Trait EI have highlighted that the HEXACO conception of extraversion is strongly correlated with Trait EI ( $r$ 's of .59 and .71).

Nonetheless, the precise meanings of both the GFP and Trait EI are a little fuzzy, and there are several key limitations with the present literature. First, researchers have not compared the relative prediction of these three levels of the personality hierarchy: GFP, domains, and facets. While the GFP overlaps substantially with Trait EI (van der Linden et al., 2017), substantial variance remains unexplained and it is unclear whether this unexplained variance also constitutes overlap with personality, albeit at lower levels of the personality hierarchy, or whether it is unique to trait EI. Second, the HEXACO model of personality has emerged as particularly popular in research and practice in recent years, yet existing research has only examined correlates with Trait EI at the domain level. This has produced results distinct from their Big 5 analogues. Yet little is known about why HEXACO produces results that are distinct from the Big 5 at the domain level, and, in particular, what role the HEXACO facets play in these differences. Third, the GFP remains a controversial concept (for a critical review, see Revelle \& Wilt, 2013) with some arguing that it reflects a substantive meta-trait (van der Linden, Dunkel, \& Petrides, 2016), and others seeing it as largely reflecting social desirability or being synonymous with extraversion (de Vries, 2011). While the GFP has typically been operationalized as the first factor of domain scores (and sometimes the first factor of items or facets), only a few studies have compared how methods for calculating the GFP influence criterion correlations (e.g., Dunkel \& Van der Linden, 2014; Van der Linden et al., 2012), and important alternative approaches have not been explored. In particular, Anglim, Morse, De Vries, MacCann, and Marty (2017) raised the possibility of scoring a Social Desirability GFP as the sum of personality items weighted by their independently derived social desirability ratings. It is 
also possible to measure Big 5 GFP using non-Big 5 personality measures by using empirically derived weights (e.g., a weighted composite of HEXACO facets). By systematically comparing the effect of GFP scoring procedures on correlations with Trait EI, a better understanding of both concepts may be obtained.

The current study sought to address these issues and thereby make three fundamental contributions to the understanding of the relationship between personality and Trait EI. First, it assessed the degree to which personality and Trait EI overlap at progressively more refined levels of the personality hierarchy: The GFP, domains, and facets. To our knowledge no previous study has examined whether facet-level effects (from any personality framework) account for the overlap between personality and Trait EI. Second, by measuring both HEXACO domains and facets, our study provides a facet-based explanation for the unique profile of domainlevel correlates observed between HEXACO personality and Trait EI. Third, by systematically varying the method of scoring the GFP, it seeks to contribute to understanding of the GFP and what drives the overlap with Trait EI. To achieve these aims, the current study had a large sample of over 1300 Australian adults complete a measure of Trait EI along with the full-length 200-item HEXACO personality inventory that is optimized for reliable facet-level measurement.

\section{Trait Emotional Intelligence}

While some researchers conceptualize EI as the ability to perceive, control, and utilize emotions (Goleman, 1995), many other researchers have focused on Trait EI represented by a constellation of affectrelated personality traits (Petrides \& Furnham, 2001; Petrides, Furnham, \& Mavroveli, 2007; Van Rooy \& Viswesvaran, 2004). Meta-analysis shows that Trait EI correlates only weakly with ability-based EI $(r=.26)$ (Joseph \& Newman, 2010). EI can be distinguished at the construct level between ability and mixed (i.e., traits and abilities) perspectives, and at the measurement level, between performance-based and self-report approaches (Joseph \& Newman, 2010). Trait EI aligns with the mixed perspective and self-report (or other-report) measurement with popular measures including the Schutte et al. (1998), the Trait Emotional Intelligence Questionnaire (TEIQue, Petrides, 1998) and the Bar-On Emotional Quotient Inventory. 


\section{Personality Traits}

Personality traits describe relatively stable individual differences in how people think, feel, and behave. They are typically conceptualized hierarchically whereby broad traits can each be understood in terms of narrower facets (for reviews, see Anglim \& O'Connor, 2019; Saucier \& Srivastava, 2015). The most popular conceptualization of broad traits in recent history has been the "Big 5" (Costa \& McCrae, 1995), but various other representations have been proposed. In particular, based on updated lexical studies, the six-factor HEXACO model (an acronym for honesty-humility, emotionality, extraversion, agreeableness, conscientiousness, and openness) has emerged as increasingly popular (Ashton et al., 2014; Ashton et al., 2004). This model most notably adds an honestyhumility factor and reconfigures variance associated with Big 5 agreeableness and neuroticism. The honestyhumility factor has been shown to be important for understanding a range of criteria especially those within the domains of antisocial/unethical behavior and cooperation (Hilbig, Kieslich, Henninger, Thielmann, \& Zettler, 2018; Lee, Ashton, Morrison, Cordery, \& Dunlop, 2008).

There is much less agreement about the precise number and nature of narrow traits (for reviews see, Anglim \& O'Connor, 2019; Paunonen \& Jackson, 2000). Nonetheless, most long-form measures of personality adopt a hierarchical orientation of facets, typically nested within domains. A growing body of research has also come to recognize the utility of facet-level analysis for the prediction of various life and work outcomes (Anglim \& Grant, 2014; Anglim, Lievens, Everton, Grant, \& Marty, 2018; Hough \& Oswald, 2008; Paunonen \& Jackson, 2000). The facet-level approach typically involves examining the degree to which personality facets provide incremental prediction of a criteria and assessing facet-criteria correlations (both zero-order and also potentially correcting for overlap with personality domains) (Anglim \& Grant, 2014; Anglim \& O'Connor, 2019).

Many personality trait researchers acknowledge the existence of higher-order personality traits that sit above the broad traits (DeYoung, 2006; Digman, 1997; Musek, 2007; Veselka et al., 2009). In particular, focus on a single general factor arose from the observation that 
Big 5 scale scores (with neuroticism coded as emotional stability) are all typically positively correlated (Digman, 1997; Musek, 2007; Van der Linden, te Nijenhuis, \& Bakker, 2010). Similarly, the first principal component of personality questionnaire items is usually substantially larger than the next component, and the loadings usually correspond to the social desirability of the items (Saucier \& Srivastava, 2015). This single factor has been labelled the General Factor of Personality (GFP), although there is active debate regarding its nature.

\section{Trait Emotional Intelligence and Personality}

Theoretically, personality and Trait EI cover overlapping domains. Personality seeks to define fundamental dimensions of thinking, feeling, and behaving, and by focusing on affect-related traits, Trait EI captures a subset of this personality domain. Many studies have reported correlations between the Big 5 and Trait EI (e.g., Petrides, Pita, \& Kokkinaki, 2007; Siegling, Furnham, \& Petrides, 2015) and two studies have reported HEXACO correlations (see Table 1). Joseph and Newman (2010) conducted a meta-analysis and obtained raw correlations of self-report mixed EI with the Big 5 ranging from .26 to .45 (see Table 1). More recently, van der Linden et al. (2017) reported metaanalytic correlations between Big 5 personality and Trait EI using the TEIQue ranging from .31 to .58 .

Empirical evidence also suggests that Trait EI is highly correlated with the GFP (Pérez-González \& Sanchez-Ruiz, 2014; van der Linden et al., 2017; Van der Linden et al., 2012) with debate focusing on whether they are synonymous constructs and on what the correlation means. First, the moderate and consistently positive correlations between the Big 5 domains (after reversing neuroticism) and Trait EI are consistent with correlations with the GFP. Second, individual studies have obtained correlations of around .70 between the GFP and Trait EI: Van der Linden et al.'s study (2012) ranged from .65 to .78 and Pérez-González and Sanchez-Ruiz (2014) obtained $r=.69$. Third, a meta-analysis conducted by van der Linden et al. (2017) found that Trait EI and the GFP had a reliability-corrected correlation of approximately .85 .

Nonetheless, given the challenges of estimating the meta-analytic correlation between Trait EI and GFP, it is unclear how much confidence should be placed in this finding of van der Linden et al. (2017). First, 
estimating the raw correlation between the GFP and Trait EI in a given study requires accurate Big 5 intercorrelations. These Big 5 inter-correlations were not available to Van der Linden et al. (2017) on a study-bystudy basis, however, and hence the authors were required to use fixed meta-analytic estimates of the Big 5 inter-correlations. In practice, however, the Big 5 intercorrelations will have varied systematically and substantially in form and overall magnitude across studies, based on measures used and sample characteristics. If multicollinearity among the Big 5 is incorrectly estimated in a meta-analysis, then the total variance in the criterion explained by the Big 5 will also be incorrect (for further discussing of meta-analytic SEM, see Landis, 2013). Second, using latent variable modelling to estimate correlations between Trait EI and the GFP rests on the accuracy of the assumptions embodied in the latent variable model. In particular, specifying the correlation between latent Trait EI and personality to only operate through latent GFP may lead to a weighting of the Big 5 that amplifies the correlation between Trait EI and the GFP. In addition, 'doubledipping' of reliability corrections may occur, as the latent variables are error-free, and therefore the shared variance may be inflated due to the double-correction. Our recommended approach for meta-analytically estimating the correlation between the GFP and Trait EI would be to estimate the correlation directly in each sample and only include samples that permit direct calculation. More generally, studies involving large samples, highly reliable measurement, and directly estimated correlations are particularly informative. For example, assuming reliability of .90 and commonly observed correlations between Trait EI and GFP of .65, .70, and .78, we obtain latent correlations of .72, .78, and .87 respectively. From this perspective, the .85 latent correlation of van der Linden et al. (2017) is plausible, but so are somewhat lower estimates which open up substantial scope for a more nuanced personality mapping.

Furthermore, examination of any incremental prediction of domains and facets over and above the GFP is relevant to assessing the degree to which Trait EI is synonymous with the GFP, and whether any remaining variance is captured by traditional personality hierarchies. Analysis of Trait EI at the facet-level is limited. While a few studies have conducted joint factor- 
analyses combining Trait EI subscales and personality facets (Pérez-González \& Sanchez-Ruiz, 2014; Petrides \& Furnham, 2001; Petrides, Pita, et al., 2007), no research to our knowledge has reported facet-level personality correlations with Trait EI. Similarly, no research has compared the relative overlap of personality and Trait EI by comparing models predicting Trait EI using personality domains and facets. Understanding the relative prediction of Trait EI by the GFP, domains, and facets is essential for understanding both the degree overlap of between personality and Trait EI, and for evaluating claims that Trait EI is largely synonymous with the GFP.

Research Question 1: To what extent does personality and Trait EI overlap and to what extent does this vary across levels from the GFP to domains to facets?

\section{HEXACO Personality and Trait EI}

To our knowledge, only two studies (Austin \& Vahle, 2016; Veselka et al., 2010) have reported correlations of HEXACO personality domains and Trait EI. The correlations from Austin and Vahle (2016) and Veselka et al. (2010) suggest both similarities and differences with the correlations seen with the Big 5 (see Table 1). First, the correlation between HEXACO extraversion and Trait EI is particularly strong. Second, the correlation between HEXACO emotionality and Trait EI is substantially lower than seen for Big 5 neuroticism. Third, the correlations of Trait EI with HEXACO agreeableness, conscientiousness, and openness are all moderate to large, consistent with a GFP-style effect. Furthermore, using the same data as Veselka et al. (2010), Veselka et al. (2009) conducted a joint factor analysis of HEXACO domains and four facets of Trait EI and labelled the first factor the GFP. In addition to all Trait EI facets loading highly, HEXACO domains loaded $.19,-.42, .73, .25, .40$, and .32 for H, E, X, A, C, and O, respectively. Importantly, existing studies have used the HEXACO 60 and have not reported facet-level correlations. It is also interesting to consider whether extraversion in the HEXACO framework largely captures the GFP. Investigation of facet-level correlates may assist in explaining why the correlations of the HEXACO domains, especially emotionality and extraversion, differ from those seen with the Big 5 . Furthermore, the lack of any systematic examination of 
facet-level correlations with Trait EI using any personality framework, makes the examination of facetlevel correlates particularly important.

Research Question 2: How do the domains and facets of the HEXACO personality framework relate to Trait EI?

\section{Measuring the GFP}

Musek (2007) suggested that the GFP may reflect an ideal score on each personality construct compared against a societal archetype and some have conceptualized the GFP as representing social effectiveness (Van der Linden et al., 2012). On the other hand, others have been critical of the concept of a GFP (Davies, Connelly, Ones, \& Birkland, 2015; de Vries, 2011; Revelle \& Wilt, 2013) with some suggesting that the GFP simply reflects social desirability bias or a blend of facets (Ashton, Lee, Goldberg, \& de Vries, 2009). Notwithstanding its nature, initial research suggests that the GFP is fairly robust to different methods of extraction (e.g., using factors or facets; for a review, see van der Linden et al., 2017). More recently, however, Anglim et al. (2017) suggested that it may be more appropriate to conceptualize and measure the GFP at the item-level using item-level social desirability weightings. In many cases where item social desirability is correlated with construct definition, the social desirability of items manifest in correlated factors and, in such cases, the GFP derived from factors and from items should be nearly identical (Anglim et al., 2017). However, for some measures, this alignment of socially desirable items and constructs may be less complete, and some samples may show less variance in social desirability. In these cases, an item-level extraction may be more robust. In general, little research has compared the consequences of this for GFP measurement and none has examined the implications for correlations with Trait EI. Indeed, though van der Linden et al. (2017) argue that the different methods of GFP extraction tends to produce highly consistent results, we are aware of only a small amount of research that assess the effect of systematically varying the method of GFP extraction (e.g., Dunkel \& Van der Linden, 2014; Van der Linden et al., 2012).

Research Question 3: To what extent does the overlap between the GFP and Trait EI vary based on how the GFP is operationalized? 


\section{Method}

Data, data analysis scripts, supplementary materials, and item-level information is provided at https://osf.io/uwdgs/

\section{Participants and Procedure}

Data were collected by an Australian organizational consultancy firm. Potential participants were sent an email inviting them to complete a confidential online survey. The invitation highlighted the benefits of the study to science and practice. Participants were also informed that one participant would receive a travel voucher. The final sample consisted of 1370 participants $(51 \%$ male, age in years $M=45.5, S D=$ 11.72, range: 21 to 71 ). The sample was somewhat more educated than then the general Australian population: $41 \%$ had a postgraduate degree and $27 \%$ had a bachelor as their highest degree. Participants worked in a broad cross-section of industries. This final sample was drawn from an initial sample of 1403 cases, where 33 cases were removed for one or more of the following reasons: (a) providing the same response to all Assessing Emotions Scale (AES) items $(n=4$; e.g., selecting "agree" for all items), (b) providing the same response to all HEXACO items $(n=3)$, and (c) taking an average of less than 3 seconds per item $(n=31)$.

\section{Measures}

HEXACO Personality. The 200-item HEXACO-PI-R is a measure of personality comprised of six domain and 25 facets (Ashton et al., 2004). Each domain score is composed of four facets, and there is also one interstitial facet titled altruism. Items were rated on a 5-point Likert scale from 1 = strongly disagree to $5=$ strongly agree. Domain and facet scales were the mean of constituent items after any item reversal.

General Factor of Personality (GFP). We derived "GFPs" in several different ways, and we assigned different labels to each derived GFP to aid distinguishability. While we use the term "GFP" as a convenient label, we acknowledge that such variables do not necessarily emanate from an objective latent general factor. Item GFP, Facet GFP, and Domain GFP were all obtained by taking the score on the first unrotated factor of a maximum likelihood factor analysis. The difference between these three GFPs lied in the variables that were included in the analysis: Item GFP was derived from the factor analysis of the 200 HEXACO-PI-R items, Facet 
GFP was derived from the 25 HEXACO facet scores, and Domain GFP was derived from the six HEXACO domain scores.

We also derived a Big 5 GFP using the HEXACO items so we could capture a GFP that was explicitly aligned with the Five Factor Model. To achieve this, we consulted the data from Horwood and Anglim's (2018) study of Australian university students who had completed the same 200 item HEXACO inventory as well as the 300 item IPIP-NEO for the purpose of obtaining feedback on their personality. In that study, a GFP was obtained for the 300 item IPIP-NEO using factors, facets, and items. Regression models were then conducted predicting these three GFPs from the 25 HEXACO facets. To generate simple and robust weights, coefficients that were significant at $p<.001$ were identified. All coefficients were divided by the absolute value of the largest coefficient, multiplied by five, and rounded. The three IPIP GFPs were almost identical: IPIP Item GFP with IPIP Facet GFP $(r=.996)$, IPIP Item GFP with IPIP Domain GFP $(r=.965)$, IPIP Facet GFP with IPIP Domain GFP $(r=$.972). In addition, the resulting coefficients were identical when predicting Facet and Item GFP and almost identical when predicting Domain GFP. As such, the Facet/Item GFP coefficients were applied to the current dataset in order to obtain Big 5 GFP. Ultimately, the data revealed that Big 5 GFP scores can be derived from the HEXACO facets by the sum of a weighted subset of HEXACO facets. The weights are as follows: Anxiety (-2), Social Self-Esteem (5), Social Boldness (3), Liveliness (3), Diligence (5), and Prudence (2). This Big 5 GFP based on weighting HEXACO facets had an adjusted multiple correlation (adjustment based on 25 facets) of $.89, .88$, and .86 with IPIP GFP using items, facets, and domains respectively.

Finally, we calculated a Social Desirability GFP, which is derived from the 200 HEXACO-PI-R items and social desirability estimates for each of the items reported by Anglim et al. (2017) (see https://osf.io/uwdgs/). Specifically, we operationalized the social desirability of each item as the Cohen's $d$ of the difference between the applicant and non-applicant samples. Social Desirability GFP scores were then calculated as the weighted-sum of individuals' responses to the items where the weights were based on the item social desirability.

Trait Emotional Intelligence. Self-reported 
Trait EI was measured using the Modified Assessing Emotions Scale (AES) (Austin, Saklofske, Huang, \& McKenney, 2004). For scoring purposes we focused on the original 33 AES items (Schutte, Malouff, \& Bhullar, 2009; Schutte et al., 1998). The modified version reversed several of the original AES items to reduce issues with having all positively worded items. We also obtained four subscales based on those recommended in the review of the AES by Schutte et al. (2009). These measured perceptions of emotions, managing own emotions, managing others' emotions, and utilization of emotions. Items were rated on a scale from $1=$ strongly disagree to $5=$ strongly agree, and scale scores were the mean of constituent items after any necessary reversal. The total score on the AES has shown strong correlations with overall scores on the TEIQue (Petrides, 1998) with correlations ranging from .73 (Gardner \& Qualter, 2010) to .83 (Stough, Saklofske, \& Parker, 2009).

\section{Data Analytic Approach}

The data analytic approach is broadly grounded in the methodology for conducting domain and facetlevel analyses described in Anglim and Grant (2014). Research Question 1 examined the relative overlap of Trait EI and personality at different levels of the trait hierarchy. This was evaluated by fitting a set of regression models predicting overall Trait EI and the EI subscales from four different sets of predictors. Model 1 included only Extraversion; Model 2 included only the GFP (specifically we used the Item GFP); Model 3 included the six HEXACO domains; and Model 4 included the 25 HEXACO facets. Interpretation focused on the adjusted r-squared values for these four models with emphasis placed on the following comparisons: (a) Extraversion versus Domains, (b) GFP versus Domains, and (c) Domains versus Facets. Adjusted r-squared provides an unbiased estimate of population variance explained. This is particularly important when comparing models with different numbers of predictors. We also report 95\% bootstrap confidence intervals on this estimate of incremental variance explained (Anglim \& Grant, 2014).

Research Question 2 examined the specific pattern of domain- and facet-level relationships of HEXACO personality with Trait EI. We assessed zeroorder correlations between domains and Trait EI. For facets, we report both zero-order and semi-partial 
correlations. The semi-partial correlations removed the overlap of each personality facet with the six HEXACO domains (i.e., saving the residual for each facet after predicting that facet from the six HEXACO domains). The square of these values indicate the percentage of variance explained in Trait EI over and above the domains. These semi-partial correlations provide an important complement to the zero-order correlations in highlighting the unique value provided by the facet; sometimes described as incremental validity. Coefficients for domain and facet-level regression models were also reported, although we place only limited emphasis on the facet-level regression coefficients given the substantial multicollinearity among facets from a common domain.

Research Question 3 examined the degree to which the overlap between Trait EI varies based on how the GFP is operationalized. This was largely assessed by comparing zero-order correlations of Trait EI with the different measure of the GFP.

More generally, because the sample size is large, correlations were estimated with a very high degree of precision. For instance, the standard error of correlations was $0.026,0.024,0.017$, and 0.005 for correlations of 0 , $.3, .6$, and .9 respectively. Thus, we focus interpretation on the key patterns, which are almost all highly significant $(p<.001)$. We used the paired. $r$ function from the psych package to compare correlations (Revelle, 2018).

\section{Preliminary Analyses}

\section{Results}

Before examining relations of Trait EI with personality, we first inspected the characteristics of the two measures. Item-level data and descriptive statistics for Trait EI are provided in the online supplement. Cronbach's alpha reliability was strong for Total EI (.87) however the reliabilities of the subscales were variable: .85 for perception of emotion, .74 for managing own emotions, .57 for managing others' emotions, and .59 for utilization of emotion. Reliability for HEXACO personality was excellent with mean alpha of .89 (range: .87 to .92 ) for HEXACO domains and .79 (range: .69 to .87) for facets. A six-factor exploratory factor analysis of the 25 HEXACO facets using maximum likelihood extraction and promax rotation showed excellent convergence to the six-factor structure with every facet 
loading maximally on its proposed domain and only two cross-loadings above .30 . The scree plot of the 25 facets also clearly supported a six-factor solution with the percentage variance explained by the first six factors being $21.0,11.6,9.6,8.0,7.2,5.5$, followed by $3.5,3.1$, 2.8, and 2.6.

\section{Correlations}

Table 2 presents the correlations between Trait EI, GFPs, and HEXACO domains. All EI subscales were positively inter-correlated but utilization of emotion showed the weakest correlations with the other subscales. In terms of the GFP, all five measures of the GFP were very similar ( $r s$ between GFPs ranged from .77 to .99 ). In particular, the GFPs based on factor analysis of items, facets, and domains were highly correlated ( $r s$ ranged from .97 to .99 ). Big 5 GFP and Social Desirability GFP also correlated strongly with the factor-analytically derived GFPs, but the correlation ( $r$ s ranged from .88 to $.94)$ were slightly weaker $(p<.001)$ and the correlation between Big 5 and Social Desirability GFP was weaker $(p<.001)$ again at $r=.77$. Examination of differential GFP correlations with HEXACO factors highlights the subtle differences. Item, Facet, and Domain GFP all showed particularly strong correlations with extraversion and then moderate correlations with all other factors (albeit, as expected, negatively with emotionality). In contrast, Social Desirability GFP weighted honestyhumility and agreeableness slightly more, and emotionality and openness somewhat less $(p<.001)$. Big 5 GFP weighted honesty-humility, agreeableness, and openness less $(p<.001)$ than the factor analytic GFPs.

GFP Correlations. Overall, Item GFP showed a very strong correlation with Total EI $(r=.62)$. At the subscale level, correlations with Item GFP was greatest for managing own emotions $(r=.72)$ followed by perception of emotions $(r=.53)$ and managing others' emotions $(r=.39)$. In contrast, in addition to utilization of emotions having only weak correlations with the other subscales, it was relatively uncorrelated with the GFPs ( $r$ s ranged from .02 to .08). Item GFP, Facet GFP, and Domain GFP all exhibited similar correlations with Total EI, whereas Social Desirability GFP showed a slightly weaker $(p<.001)$ correlation with Total EI $(r=.53)$. In general, the patterns of correlations with EI subscales for the different GFPs was very similar.

Domain correlations. Of the HEXACO domain 
scores, extraversion was clearly the strongest correlate of Total EI $(r=.67)$, although openness $(r=.39)$, conscientiousness $(r=.35)$, and agreeableness $(r=.26)$ all had noteworthy correlations. Perception of emotions largely mirrored this pattern and managing own emotions was also similar, except that its negative correlation with emotionality was noticeably larger in size $(r=-32 ; p<$ $.001)$. Managing others' emotions differed from the above pattern by its positive correlation with emotionality. Utilization of emotion showed a fairly distinct pattern of correlations in that it was most strongly associated with emotionality $(r=.27)$ and openness $(r=$ .31) with only small correlations with the other domains.

Facet correlations. Table 3 presents the zeroorder and residualized correlations (i.e., where variance overlapping with HEXACO domains is removed from the facet) of the HEXACO facets with Trait EI. The strongest zero-order correlates of Total EI were the extraversion facets of social self-esteem $(r=.59)$ and liveliness $(r=.60)$. The residualized correlations highlight the differential relationship of extraversion and emotionality facets with Total EI. Specifically, sentimentality was positively related to Total EI, but the other facets were negatively related to Total EI. In relation to extraversion, sociability was slightly negatively related to Total EI after controlling for the six HEXACO domain scores. The interstitial trait of altruism was a strong incremental predictor of Total EI. The correlations with dependence and sentimentality were particularly prominent for managing others' emotions and utilization of emotion.

\section{Regression Models}

Table 4 shows the adjusted $R$-squared for regression models predicting Trait EI from the four predictor sets: (a) extraversion, (b) Item GFP, (c) the HEXACO domains, and (d) the 25 HEXACO facets. Table 4 also reports the difference in variance explained for the three model comparisons of primary interest: Extraversion versus Domains, GFP versus Domains, and Domains versus Facets. When predicting Total EI, HEXACO domains (adjusted $R^{2}=.55$ for HEXACO Domains) explained more variance than either Extraversion (Adjusted $R^{2}=.44 ; \Delta$ adjusted $R^{2}=.11$, $95 \%$ CI $[.08, .13]$ ) or the GFP (Adjusted $R^{2}=.39 ; \Delta$ adjusted $R^{2}=.16,95 \%$ CI $\left.[.13, .20]\right)$. HEXACO facets (Adjusted $R^{2}=.65$ ) also explained additional variance 
over and above HEXACO Domains ( $\Delta$ adjusted $R^{2}=.10$, $95 \%$ CI $[.08, .12])$. In terms of subscales, perceptions of emotions showed a similar pattern with generally reduced correlations. Managing own emotions showed a smaller increase from GFP to domains. Managing others' emotions was poorly predicted by the GFP and extraversion, but was still reasonably well predicted by domains and facets. Finally, utilization of emotion was relatively poorly predicted in general and was unrelated to the GFP.

Table 5 presents the standardized coefficients for the regression model with the six HEXACO domains (see online supplement for facet-level regression coefficients). In relation to Total EI, extraversion was the strongest predictor, but openness, conscientiousness, and emotionality were also moderately strong predictors. The positive standardized regression coefficient for emotionality $(\beta=.20)$ contrasted with its zero-order correlation, which was close to zero $(r=-.03)$. Examination of Trait EI subscales suggests that extraversion was a strong predictor for perceptions of emotion, managing own emotions, and managing others' emotions, emotionality was a strong predictor of managing others' emotions and utilization of emotion, and openness was a strong predictor of utilization of emotion.

\section{Discussion}

The current study aimed to systematically examine the overlap between personality and Trait EI focusing first on the GFP, derived in several different ways, and then at the domain- and facet-levels of the HEXACO personality model. Several key findings emerged. First, all methods for deriving the GFP with the HEXACO-PI-R correlated very highly with one another. Second, Item GFP, Facet GFP, and Big 5 GFP showed the strongest correlations with Trait EI ( $r=.62$ to .64$)$. Third, HEXACO extraversion correlated slightly stronger with Trait EI than did the GFP, notwithstanding the derivation of the latter. Fourth, personality domainlevel regression models led to improved prediction of Trait EI over and above the GFP, where the best composite heavily weighted extraversion and moderately weighted emotionality, conscientiousness, and openness. Fifth, personality facet-level regression models improved predictions of Trait EI over and above domains. In particular, altruism, sentimentality, and social self- 
esteem were relatively strong facet-level predictors of Trait EI. Altogether, the results have implications for the understanding of the overlap between personality and Trait EI and the conceptualization of the GFP.

\section{Overlap between Trait EI and Personality at Different Levels}

While good approximations of Trait EI can be obtained by both the GFP $(r=.62)$ and HEXACO extraversion $(r=.67)$, substantial gains in prediction can be achieved by appropriately weighting domains (adjusted multiple $r=.74$ ) and facets (adjusted multiple $r=.81$ ). Gains at facet-level were particularly noteworthy. Thus, Trait EI appears not to be synonymous with the GFP. Our results suggest a large part of the variance not accounted for by the GFP does constitute overlap with personality, albeit at lower levels of the personality hierarchy. Trait EI is multifaceted and well captured by a hierarchical framework of personality such as the HEXACO. The core of the construct aligns closely with the GFP, but it has other elements such as openness to emotions and placing importance on emotional experience. Optimal prediction of Total EI involved greater weighting of extraversion and less weighting of agreeableness and honesty-humility than would be implied by the GFP. Emotionality was also positively related to Trait EI after controlling for other HEXACO domains.

While Trait EI is largely captured by personality (Conte, 2005; Daus \& Ashkanasy, 2003), this overlap is best captured at the facet-level. Facet-level regression yielded substantial improvements in prediction beyond the domain-level (approximately 20\% better than domains; and $65 \%$ better than the GFP). This was largely caused by differential prediction across facets within domains, particularly with respect to extraversion, emotionality, and openness. Sentimentality $(+)$, social self-esteem $(+)$, sociability $(-)$, inquisitiveness $(-)$ and creativity $(+)$ provided incremental prediction over and above the domains. These facets better captured the essence of Trait EI combining the importance of emotions with well-being and social effectiveness. The interstitial facet of altruism also incrementally predicted Trait EI. This facet captures a general concern for the well-being of others and unsurprisingly it correlated best with managing others' emotions.

Our findings, suggesting that personality facets 
are largely able to capture Trait EI, differ in emphasis from the few previous studies that have examined personality facets and Trait EI. Previous research has analyzed data on personality facets and Trait EI by conducting joint exploratory factor analyses of personality facets (e.g., the NEO-PI-R) and Trait EI subscales (i.e., Pérez-González \& Sanchez-Ruiz, 2014; Petrides \& Furnham, 2001; Petrides, Pita, et al., 2007). They have reported six factor solutions indicating that many Trait EI subscales load on their own factor and the Big 5 facets generally load on the remaining five factors. For example, Pérez-González and Sanchez-Ruiz (2014) concluded that Trait EI is a distinct and unique factor in the Big 5 factor space. However, we believe that the correlational and regression based approach used in the current study is more appropriate for assessing the novelty of Trait EI. In particular, Trait EI does not align with any one personality domain. Rather the common variance of Trait EI is a composite of several domains and facets. As such, it is unsurprising that Trait EI subscales do not load on any one personality domain. Rather Trait EI is largely a composite of multiple personality facets and domains, which, while similar to the GFP (itself a multi-domain composite), incorporates a unique profile of domains and facets.

\section{HEXACO Domains and Facets and Trait EI}

Correlations between Trait EI and the six HEXACO domains were similar to those obtained by the two previous studies that employed the HEXACO model (Austin \& Vahle, 2016; Veselka et al., 2010). Extraversion was by far the strongest correlate; agreeableness, conscientiousness, and openness all showed correlations with Trait EI in the .30 to .40 range; honesty-humility had a weak positive, and emotionality had a weak negative correlation with Trait EI. The weakness of the negative correlation of emotionality is closer to the results of Austin and Vahle (2016) than to those of Veselka et al. (2010) (see Table 1).

The present study and the two other HEXACO studies highlight differences with Big Five meta-analytic results (Joseph \& Newman, 2010; van der Linden et al., 2017). The most prominent differences are that correlations with HEXACO extraversion are much larger than for Big 5 Extraversion, and correlations for emotionality are much smaller than for neuroticism. Thus, it is the particular configuration of Big 5 variance, 
rather than the addition of honesty-humility, which gives the HEXACO its unique correlational profile in relation to Trait EI. Results from Gaughan, Miller, and Lynam (2012) mapping NEO-PI-R and HEXACO-PI-R are particularly informative. Specifically, these analyses show that while HEXACO extraversion correlates highly with NEO extraversion $(r=.86)$, the former also exhibits a relatively large negative correlation with NEO neuroticism $(r=-.50)$. In contrast, the correlation between HEXACO emotionality and NEO neuroticism is modest $(r=.52)$ for two scales which might superficially appear to be analogous. An examination of facet content and facet-level correlations with Trait EI further helps to explain these differences.

The correlations between HEXACO extraversion and Total EI was particularly strong $(r=.67)$ and consistent with the two previous studies that used the HEXACO model (Austin \& Vahle, 2016; Veselka et al., 2010). HEXACO extraversion also correlated more with overall Trait EI than the GFP. Facet-level correlations help to explain why this is so. Specifically, social selfesteem and liveliness exhibited particularly strong correlations with Trait EI, whereas sociability showed a weaker correlation that was slightly negative, when controlling for domain scores. Liveliness measures a positive and optimistic orientation, which is almost synonymous with some components of Trait EI, and this is reflected in the .70 correlation with managing own emotions. Social self-esteem measures the tendency to feel positive self-regard, particularly in social contexts. Thus, it comes closest to a general well-being factor which is also closely related to some facets of Trait EI. Overall, relative to some Big 5 measures of extraversion, the HEXACO conception of extraversion captures more well-being and optimism - all core components of Trait EI.

HEXACO emotionality differs from neuroticism in several ways. The essence of neuroticism is a tendency to experience negative emotions including stress, anxiety, and depression. It often incorporates maladaptive coping strategies and may include other negative emotions, including anger and hostility. In contrast, while HEXACO includes the facet of anxiety (and to a lesser extent fearfulness) that comes closest to the essence of neuroticism, it also includes the quite different facets of sentimentality and dependence, which 
are more typically characteristic of Big 5 agreeableness (Ashton et al., 2014). Unsurprisingly, the facet of anxiety showed a negative correlation with Total EI $(r=-.24)$ and it was particularly strongly related to an inability to manage one's own emotions $(r=-.46)$. However, the facet of sentimentality which captures the tendency to feel strong emotional bonds with others actually had a strong positive correlation with Trait EI $(r=.38)$, managing others' emotions $(r=.48)$, and utilization of emotions $(r=.36)$. While Trait EI does emphasize the adaptive use of emotions, a component of this is placing some importance on emotions. Emotionality, and the sentimentality facet in particular, gets closer to this conceptualization. This also helps to explain why emotionality had a weak negative correlation with Trait EI, but its standardized regression coefficient in a regression that includes all six HEXACO domains predicting Trait EI was positive. Presumably, the other broad traits removed much of the variance related to tendencies to experience negative emotions, and what remained was a purer measure of a tendency to experience and value emotions.

\section{Implications for GFP Measurement}

The correlations between the GFP and Total EI speak to the meaning of both constructs. The correlations between Item GFP and Trait EI in the current study ( $r=$ .62) were similar to those obtained in Van der Linden et al. (2012) ranging from .65 to .78 and Pérez-González and Sanchez-Ruiz (2014) who obtained $r=.69$. First, correlations between Total EI and the GFP were greater for Item GFP than for Social Desirability GFP. The essence of Trait EI is social effectiveness, optimism, and well-being; characteristics that are better captured by HEXACO extraversion than by traits emphasized more by social desirability GFP (e.g., honesty-humility, agreeableness). The slightly stronger correlations for Item GFP than for Domain GFP may also reflect the more refined estimate that can be obtained from items.

The present research scored the GFP in five different ways, and thereby speaks to other studies that have compared GFP extraction using different measures and methods of extraction (Dunkel \& Van der Linden, 2014; Van der Linden et al., 2012). All approaches were strongly intercorrelated. That said, the correlations of the Social Desirability GFP and Big 5 GFP with factor analytic GFPs and especially with each other were 
somewhat smaller. Correlations between GFPs and HEXACO domains highlights the subtle differences. Specifically, Item, Facet, Domain and Big 5 GFPs all placed particular emphasis on extraversion. Big 5 GFP reduced the emphasis on honesty-humility, agreeableness, and openness. The greater importance of honesty-humility and agreeableness in HEXACO GFPs mirrors the greater importance placed on these domains in the HEXACO model. The Five Factor Model generally situates honesty-humility and the non-anger components of HEXACO agreeableness in its agreeableness factor. This also highlights how the extracted GFP can be slightly altered by the particular substantive focus of a personality inventory. In contrast, Social Desirability GFP placed greater emphasis on honesty-humility and agreeableness and less emphasis on emotionality and openness. In particular, honesty-humility and HEXACO agreeableness are seen as social desirable qualities (Anglim et al., 2017), but when derived from the first factor, the GFP seems to be drawn more towards optimism, interpersonal effectiveness, and well-being. This highlights a subtle distinction whereby the GFP may be better conceptualized in terms of social effectiveness, self-efficacy, optimism, and well-being and embodying less the morality and integrity components of social desirability.

In theory, GFPs based on items should be more robust across different personality questionnaires because it is possible to remove correlations between factors through various item writing and test development practices (Bäckström, Björklund, \& Larsson, 2009). In particular, openness and emotionality domains in the HEXACO model are more balanced in relation to social desirability (Anglim et al., 2017), but this is partially achieved by having a mix of positive and negative facets. Furthermore, in practice, many popular research measures of personality (e.g., HEXACO-PI-R, NEO-PI-R, IPIP) tend to have scales that are fairly uniform in their social desirability orientation. As such, the GFP based on items will tend to converge with the GFP based on scales.

Such a convergence is further contingent on the degree to which the population varies in the tendency to evaluate items positively or negatively. This is typically indexed by the size of the variance explained by the first factor and by the pattern of factor correlations. For 
instance, evaluative variance and factor correlations in low-stakes HEXACO 200 studies appears to have been particularly low in the Dutch sample of de Vries (2011), moderate in the United States sample of Gaughan et al. (2012), and somewhat higher in the current study (an Australian community sample) and the Australian university sample of Horwood and Anglim (2018). If a subset of participants engages in positive spin or deliberate faking, evaluative variance will increase. However, there may also be substantive factors which lead some samples to vary more in the actual social desirability of personality traits. More research is needed to understand the causes of variation in evaluative variance across low-stakes studies.

The results also highlight how HEXACO extraversion aligns more closely with the GFP than on other personality scales. HEXACO extraversion appears to contain more well-being and reversed neuroticism than Big 5 equivalents along with the usual social effectiveness components, which together represent key aspects of the GFP. Nonetheless, this study provides an empirically based method for scoring the HEXACO facets if a Big 5-style GFP is desired (see Method section). While this weights extraversion facets highly, it also includes facets of conscientiousness $(+)$ and the emotionality facet of anxiety (-).

It seems likely that the GFP is largely a formative construct rather than a reflective construct, and that between-person variance in social desirability bias makes it appear like a reflective construct. From this perspective, correlations between personality domains largely emerge from item-level social desirability bias (creating an apparent reflective factor). However, at the same time, these correlations also define a method for creating a composite that may actually be desirable (a formative factor). Socially desirable traits are socially desirable for a reason. Thus, to be truly high on these traits is likely to provide benefits in that a person is likely to be more socially effective, happier, and hard working. Thus, if traits like extraversion, emotional stability, and conscientiousness are truly uncorrelated, forming a weighted composite of these three variables might be a practical predictor of a range of important criteria such as well-being and interpersonal effectiveness. Such a perspective would also reconcile research based on selfother ratings which suggest that much of the correlation 
between personality domains is due to halo bias (DeYoung, 2006) yet item-level evaluative factors show strong self-other correlations (Chen, Watson, Biderman, \& Ghorbani, 2016).

\section{Limitations}

Consistent with the broader literature studying Trait EI and personality, the current study used selfreport assessments. This limits the confidence regarding generalization to the underlying objective constructs. In particular, any evaluative variance that reflects social desirability bias rather than substance may elevate the correlations between personality and Trait EI. Future research should consider alternative measurement approaches including other-reports and behavioral observation, which will enable assessment of whether the GFP is a general factor of objective personality as opposed to a general factor of self-report ratings. Nonetheless, the current research is particularly relevant to researchers and practitioners seeking to understand the overlap of self-report Trait EI and personality measures.

\section{Conclusion}

Using a large sample and full-length measures, the current research makes several fundamental contributions to research on personality, Trait EI, and the GFP. It provides the first comprehensive facet-level analysis of the correlates of personality and Trait EI. The precision achieved with the large sample and the facetlevel analysis provide great insight into the unique profile of correlations between HEXACO domains and Trait EI. The research also contributes to fundamental discussion about the relationship between Trait EI and personality showing how domains and facets can achieve superior prediction to the GFP. The research also shows that Trait EI is well captured by comprehensive hierarchical measures of personality.

\section{Declaration of Conflicting Interests}

The fifth author uses measures of HEXACO personality as part of his consulting business. Otherwise, the authors declared no potential conflicts of interest with respect to the research, authorship, and/or publication of this article.

\section{Funding}

The authors received no financial support for the research, authorship, or publication of this article. 


\section{References}

Anglim, J., \& Grant, S. L. (2014). Incremental criterion prediction of personality facets over factors: Obtaining unbiased estimates and confidence intervals. Journal of Research in Personality, 53, 148-157.

Anglim, J., Lievens, F., Everton, L., Grant, S. L., \& Marty, A. (2018). HEXACO personality predicts counterproductive work behavior and organizational citizenship behavior in low-stakes and job applicant contexts. Journal of Research in Personality, 77, 11-20.

Anglim, J., Morse, G., De Vries, R. E., MacCann, C., \& Marty, A. (2017). Comparing job applicants to non-applicants using an item-level bifactor model on the hexaco personality inventory. European journal of personality, 31, 669-684.

Anglim, J., \& O'Connor, P. (2019). Measurement and research using the Big Five, HEXACO, and narrow traits: A primer for researchers and practitioners. Australian Journal of Psychology, 71, 16-25.

Ashton, M. C., Lee, K., \& De Vries, R. E. (2014). The HEXACO Honesty-Humility, Agreeableness, and Emotionality factors: A review of research and theory. Personality and Social Psychology Review, 18, 139-152.

Ashton, M. C., Lee, K., Goldberg, L. R., \& de Vries, R. E. (2009). Higher order factors of personality: Do they exist? Personality and Social Psychology Review, 13, 79-91.

Ashton, M. C., Lee, K., Perugini, M., Szarota, P., De Vries, R. E., Di Blas, L., . . . De Raad, B. (2004). A six-factor structure of personality-descriptive adjectives: solutions from psycholexical studies in seven languages. Journal of Personality and Social Psychology, 86, 356-366.

Austin, E. J., Saklofske, D. H., Huang, S. H. S., \& McKenney, D. (2004). Measurement of trait emotional intelligence: testing and cross-validating a modified version of Schutte et al.'s (1998) measure. Personality and Individual Differences, 36 , $555-562$.

Austin, E. J., \& Vahle, N. (2016). Associations of the Managing the Emotions of Others Scale (MEOS) with HEXACO personality and with trait emotional intelligence at the factor and facet level. Personality \& Individual Differences, 94, 348-353.

Bäckström, M., Björklund, F., \& Larsson, M. R. (2009). Five-factor inventories have a major general factor related to social desirability which can be reduced by framing items neutrally. Journal of Research in Personality, 43, 335-344.

Chen, Z., Watson, P. J., Biderman, M., \& Ghorbani, N. (2016). Investigating the Properties of the General Factor (M) in Bifactor Models Applied to Big Five or HEXACO Data in Terms of Method or Meaning. Imagination, Cognition \& Personality, 35, 216-243.

Conte, J. M. (2005). A review and critique of emotional intelligence measures. Journal of Organizational Behavior, 26, 433-440.

Costa, P. T., \& McCrae, R. R. (1995). Domains and facets: Hierarchical personality assessment using the Revised NEO Personality Inventory. Journal of Personality Assessment, 64, 21-50.

Daus, C. S., \& Ashkanasy, N. M. (2003). Will the real emotional intelligence please stand up. On deconstructing the emotional intelligence "debate." The Industrial-Organizational Psychologist, 41, 69-72.

Davies, S. E., Connelly, B. S., Ones, D. S., \& Birkland, A. S. (2015). The General Factor of Personality: The "Big One," a selfevaluative trait, or a methodological gnat that won't go away? Personality and Individual Differences, 81, 13-22.

de Vries, R. E. (2011). No evidence for a General Factor of Personality in the HEXACO Personality Inventory. Journal of Research in Personality, 45, 229-232.

DeYoung, C. G. (2006). Higher-order factors of the Big Five in a multi-informant sample. Journal of Personality and Social Psychology, 91, 1138-1151.

Digman, J. M. (1997). Higher-order factors of the Big Five. Journal of Personality and Social Psychology, 73, $1246-1256$.

Dunkel, C. S., \& Van der Linden, D. (2014). Evidence for the general factor of personality as social-effectiveness. Personality and Individual Differences, 64, 147-151.

Gardner, K. J., \& Qualter, P. (2010). Concurrent and incremental validity of three trait emotional intelligence measures. Australian Journal of Psychology, 62, 5-13.

Gaughan, E. T., Miller, J. D., \& Lynam, D. R. (2012). Examining the utility of general models of personality in the study of psychopathy: A comparison of the HEXACO-PI-R and NEO PI-R. Journal of personality disorders, 26, 513-523.

Goleman, D. (1995). Emotional intelligence. New York, NY, England: Bantam Books, Inc.

Hilbig, B. E., Kieslich, P. J., Henninger, F., Thielmann, I., \& Zettler, I. (2018). Lead Us (Not) into Temptation: Testing the Motivational Mechanisms Linking Honesty-Humility to Cooperation. European journal of personality, 32, 116-127.

Horwood, S., \& Anglim, J. (2018). Personality and problematic smartphone use: A facet-level analysis using the Five Factor Model and HEXACO frameworks. Computers in Human Behavior, 85, 349-359.

Hough, L. M., \& Oswald, F. L. (2008). Personality testing and industrial-organizational psychology: Reflections, progress, and prospects. Industrial and Organizational Psychology, 1, 272-290.

Joseph, D. L., \& Newman, D. A. (2010). Emotional intelligence: An integrative meta-analysis and cascading model. Journal of Applied Psychology, 95, 54-78.

Landis, R. S. (2013). Successfully combining meta-analysis and structural equation modeling: Recommendations and strategies. Journal of Business and Psychology, 28, 251-261.

Lee, K., Ashton, M. C., Morrison, D. L., Cordery, J., \& Dunlop, P. D. (2008). Predicting integrity with the HEXACO personality model: Use of self-and observer reports. Journal of Occupational and Organizational Psychology, 81, 147-167.

Musek, J. (2007). A general factor of personality: Evidence for the Big One in the five-factor model. Journal of Research in Personality, 41, 1213-1233.

Paunonen, S. V., \& Jackson, D. N. (2000). What is beyond the big five? Plenty! Journal of personality, 68, 821-835. 
Pérez-González, J. C., \& Sanchez-Ruiz, M.-J. (2014). Trait emotional intelligence anchored within the Big Five, Big Two and Big One frameworks. Personality and Individual Differences, 65, 53-58.

Petrides, K. V. (1998). Trait Emotional Intelligence Questionnaire: London Psychometric Laboratory.

Petrides, K. V., \& Furnham, A. (2001). Trait emotional intelligence: Psychometric investigation with reference to established trait taxonomies. European journal of personality, 15, 425-448.

Petrides, K. V., Furnham, A., \& Mavroveli, S. (2007). Trait emotional intelligence: Moving forward in the field of EI. In G. Matthews, M. Zeidner, R. D. Roberts, G. Matthews, M. Zeidner, \& R. D. Roberts (Eds.), The science of emotional intelligence: Knowns and unknowns. (pp. 151-166). New York, NY, US: Oxford University Press.

Petrides, K. V., Pérez-González, J. C., \& Furnham, A. (2007). On the criterion and incremental validity of trait emotional intelligence. Cognition and Emotion, 21, 26-55.

Petrides, K. V., Pita, R., \& Kokkinaki, F. (2007). The location of trait emotional intelligence in personality factor space. British Journal of Psychology, 98, 273-289.

Revelle, W. (2018). psych: Procedures for Personality and Psychological Research. Northwestern University, Evanston, Illinois, USA.

Revelle, W., \& Wilt, J. (2013). The general factor of personality: A general critique. Journal of Research in Personality, 47, 493504.

Saucier, G., \& Srivastava, S. (2015). What makes a good structural model of personality? Evaluating the Big Five and alternatives. Handbook of personality and social psychology, 3, 283-305.

Schutte, N. S., Malouff, J. M., \& Bhullar, N. (2009). The Assessing Emotions Scale. In C. Stough, D. H. Saklofske, J. D. A. Parker, C. Stough, D. H. Saklofske, \& J. D. A. Parker (Eds.), Assessing emotional intelligence: Theory, research, and applications. (pp. 119-134). New York, NY, US: Springer Science + Business Media.

Schutte, N. S., Malouff, J. M., Hall, L. E., Haggerty, D. J., Cooper, J. T., Golden, C. J., \& Dornheim, L. (1998). Development and validation of a measure of emotional intelligence. Personality and Individual Differences, 25, 167-177.

Siegling, A. B., Furnham, A., \& Petrides, K. V. (2015). Trait emotional intelligence and personality: Gender-invariant linkages across different measures of the Big Five. Journal of Psychoeducational Assessment, 33, 57-67.

Stough, C., Saklofske, D. H., \& Parker, J. D. A. (2009). Assessing emotional intelligence: Theory, research, and applications. New York, NY, US: Springer Science.

van der Linden, D., Dunkel, C. S., \& Petrides, K. V. (2016). The General Factor of Personality (GFP) as social effectiveness: Review of the literature. Personality and Individual Differences, 101, 98-105.

van der Linden, D., Pekaar, K. A., Bakker, A. B., Schermer, J. A., Vernon, P. A., Dunkel, C. S., \& Petrides, K. V. (2017). Overlap between the general factor of personality and emotional intelligence: A meta-analysis. Psychological Bulletin, 143, 36-52.

Van der Linden, D., te Nijenhuis, J., \& Bakker, A. B. (2010). The general factor of personality: A meta-analysis of Big Five intercorrelations and a criterion-related validity study. Journal of Research in Personality, 44, 315-327.

Van der Linden, D., Tsaousis, I., \& Petrides, K. V. (2012). Overlap between General Factors of Personality in the Big Five, Giant Three, and trait emotional intelligence. Personality \& Individual Differences, 53, 175-179.

Van Rooy, D. L., \& Viswesvaran, C. (2004). Emotional intelligence: A meta-analytic investigation of predictive validity and nomological net. Journal of vocational Behavior, 65, 71-95.

Veselka, L., Petrides, K. V., Schermer, J. A., Cherkas, L. F., Spector, T. D., \& Vernon, P. A. (2010). Phenotypic and genetic relations between the HEXACO dimensions and trait emotional intelligence. Twin Research and Human Genetics, 13, 66-71.

Veselka, L., Schermer, J. A., Petrides, K. V., Cherkas, L. F., Spector, T. D., \& Vernon, P. A. (2009). General factor of personality: Evidence from the HEXACO model and a measure of trait emotional intelligence. Twin Research and Human Genetics, 12, 420-424.

Zeidner, M., Roberts, R. D., \& Matthews, G. (2008). The science of emotional intelligence: Current consensus and controversies. European Psychologist, 13, 64-78. 
Table 1

Correlations of HEXACO and Big Five Personality with Trait EI

\begin{tabular}{llllllll}
\hline Study & $n$ & $\mathrm{H}$ & $\mathrm{E} /$ & $\mathrm{X}$ & $\mathrm{A}$ & $\mathrm{C}$ & $\mathrm{O}$ \\
& & $\mathrm{N}$ & & & & \\
\hline Big Five & & & & & & & \\
$\quad$ Joseph and Newman (2010) & $5386-5591$ & & -.45 & .27 & .36 & .33 & .26 \\
$\quad$ van der Linden et al. (2017) & 22 studies & & -.58 & .47 & .37 & .40 & .31 \\
HEXACO & & & & & & & \\
$\quad$ Veselka et al. (2010) & 2384 & .12 & -.28 & .59 & .22 & .30 & .22 \\
$\quad$ Austin and Vahle (2016) & 380 & -13 & -.17 & .71 & .39 & .48 & .22 \\
\hline
\end{tabular}

Note. $\mathrm{H}=$ honesty-humility; $\mathrm{E} / \mathrm{N}=$ emotionality / neuroticism; $\mathrm{X}=$ extraversion; $\mathrm{A}=$ agreeableness; $\mathrm{C}=$ conscientiousness; $\mathrm{O}=$ openness to experience. Sample size for TEIQue is not specified in van der Linden et al. (2017), but median sample size per study is 232, which leads to an estimate of approximately 5,000 cases. 
Table 2

Bivariate Correlations of HEXACO Domains, GFP, and Emotional Intelligence Variables

\begin{tabular}{|c|c|c|c|c|c|c|c|c|c|c|c|c|c|c|c|}
\hline Variable & 1 & 2 & 3 & 4 & 5 & 6 & 7 & 8 & 9 & 10 & 11 & 12 & 13 & 14 & 15 \\
\hline \multicolumn{16}{|l|}{ Trait EI } \\
\hline \multicolumn{16}{|l|}{ 1. Total EI } \\
\hline 2. Perception of Emotions & .84 & & & & & & & & & & & & & & \\
\hline 3. Managing Own Emotions & .78 & .53 & & & & & & & & & & & & & \\
\hline 4. Managing Others' Emotions & .76 & .52 & .44 & & & & & & & & & & & & \\
\hline 5. Utilization of Emotions & .54 & .24 & .27 & .37 & & & & & & & & & & & \\
\hline \multicolumn{16}{|l|}{ GFP } \\
\hline 6. Item GFP & .62 & .53 & .72 & .39 & .06 & & & & & & & & & & \\
\hline 7. Facet GFP & .64 & .53 & .74 & .41 & .08 & .99 & & & & & & & & & \\
\hline 8. Domain GFP & .59 & .49 & .70 & .39 & .06 & .97 & .98 & & & & & & & & \\
\hline 9. Big 5 GFP & .63 & .52 & .76 & .37 & .07 & .91 & .92 & .88 & & & & & & & \\
\hline 10. Social Desirability GFP & .53 & .47 & .61 & .36 & .02 & .94 & .92 & .92 & .77 & & & & & & \\
\hline \multicolumn{16}{|l|}{ HEXACO Domains } \\
\hline 11. Honesty-humility & .12 & .13 & .12 & .07 & .01 & .42 & .36 & .35 & .18 & .59 & & & & & \\
\hline 12. Emotionality & -.03 & -.08 & -.32 & .21 & .27 & -.49 & -.49 & -.51 & -.50 & -.32 & -.02 & & & & \\
\hline 13. Extraversion & .67 & .53 & .68 & .52 & .16 & .78 & .82 & .80 & .86 & .66 & .07 & -.27 & & & \\
\hline 14. Agreeableness & .26 & .21 & .33 & .21 & -.06 & .64 & .64 & .68 & .38 & .74 & .33 & -.23 & .32 & & \\
\hline 15. Conscientiousness & .35 & .31 & .44 & .18 & .02 & .55 & .54 & .56 & .59 & .55 & .18 & -.18 & .32 & .20 & \\
\hline 16. Openness & .39 & .28 & .32 & .29 & .31 & .38 & .39 & .42 & .27 & .25 & .05 & -.13 & .27 & .16 & .10 \\
\hline
\end{tabular}

Notes. $N=1370 .|r| \geq$ to $.06, .07$, and .09 are statistically significant at $.05, .01$, and .001 , respectively. 
Table 3

Bivariate Correlations between HEXACO Facets and Emotional Intelligence Factors

\begin{tabular}{|c|c|c|c|c|c|c|c|c|c|c|}
\hline & \multicolumn{5}{|c|}{ Zero-order correlations } & \multicolumn{5}{|c|}{ Semi-Partial Correlations } \\
\hline & Total & & & & & Total & & & & \\
\hline & EI & Perc. & Own & Others & Use & EI & Perc. & Own & Others & Use \\
\hline H1: Sincerity & .09 & .12 & .11 & .00 & -.02 & -.01 & .02 & -.02 & -.02 & -.01 \\
\hline H2: Fairness & .24 & .19 & .25 & .18 & .06 & -.01 & -.05 & .01 & .01 & .01 \\
\hline H3: Greed-Avoidance & -.01 & .00 & -.01 & -.02 & -.02 & -.04 & -.05 & -.02 & -.03 & -.03 \\
\hline H4: Modesty & .07 & .10 & .02 & .06 & .01 & .07 & .09 & .03 & .04 & .02 \\
\hline E1: Fearfulness & -.17 & -.13 & -.27 & -.04 & .00 & -.06 & -.01 & .03 & -.12 & -.16 \\
\hline E2: Anxiety & -.24 & -.24 & -.46 & -.03 & .19 & -.07 & -.07 & -.10 & -.05 & .05 \\
\hline E3: Dependence & .02 & -.07 & -.21 & .24 & .25 & -.05 & -.09 & -.05 & .01 & .02 \\
\hline E4: Sentimentality & .38 & .28 & .10 & .48 & .36 & .22 & .20 & .12 & .19 & .12 \\
\hline X1: Social Self-Esteem & .59 & .49 & .66 & .37 & .10 & .10 & .10 & .16 & -.03 & .01 \\
\hline X2: Social Boldness & .52 & .43 & .50 & .39 & .16 & -.02 & .01 & -.11 & .01 & .05 \\
\hline X3: Sociability & .42 & .32 & .31 & .48 & .14 & -.13 & -.10 & -.18 & .01 & -.10 \\
\hline X4: Liveliness & .60 & .46 & .70 & .40 & .12 & .09 & .01 & .18 & .01 & .05 \\
\hline A1: Forgiveness & .23 & .17 & .26 & .23 & -.01 & -.01 & -.03 & -.03 & .04 & .02 \\
\hline A2: Gentleness & .12 & .06 & .15 & .14 & -.02 & -.02 & -.06 & .00 & .01 & .03 \\
\hline A3: Flexibility & .18 & .19 & .23 & .11 & -.08 & -.03 & .03 & -.03 & -.08 & -.06 \\
\hline A4: Patience & .27 & .24 & .38 & .16 & -.09 & .06 & .07 & .06 & .02 & .01 \\
\hline $\mathrm{C} 1$ : Organization & .21 & .19 & .24 & .11 & .03 & -.02 & -.01 & -.06 & .00 & .04 \\
\hline C2: Diligence & .39 & .29 & .52 & .19 & .09 & .05 & -.03 & .10 & .03 & .10 \\
\hline C3: Perfectionism & .19 & .16 & .16 & .14 & .08 & -.01 & .00 & -.06 & .03 & .02 \\
\hline C4: Prudence & .24 & .26 & .39 & .07 & -.17 & -.03 & .04 & .03 & -.06 & -.17 \\
\hline O1: Aesthetic Appreciation & .31 & .22 & .20 & .28 & .25 & -.01 & .00 & -.02 & .02 & -.07 \\
\hline $\mathrm{O} 2$ : Inquisitiveness & .22 & .17 & .24 & .12 & .11 & -.12 & -.08 & -.05 & -.11 & -.14 \\
\hline O3: Creativity & .42 & .30 & .36 & .30 & .30 & .10 & .07 & .08 & .07 & .11 \\
\hline O4: Unconventionality & .24 & .15 & .16 & .16 & .28 & .04 & .01 & .00 & .02 & .12 \\
\hline I: Altruism & .44 & .31 & .27 & .46 & .31 & .18 & .11 & .12 & .19 & .16 \\
\hline
\end{tabular}

Note. $|r| \geq .06, .07$, and .09 are statistically significant at $.05, .01$, and .001 , respectively. Perc. $=$ Perception of emotion, Own = Managing own emotions, Others = Managing others' emotions, Use $=$ Utilization of emotion. Semi-partial correlations control for overlap between facet and the six HEXACO domains. Absolute zero-order correlations greater than or equal to .30 and absolute semi-partial correlations greater than or equal to .10 are bolded. 
Table 4

Adjusted $R^{2}$ for Regression Models Predicting Trait EI Factors from Various Sets of Predictors Based on HEXACO Personality.

\begin{tabular}{|c|c|c|c|c|c|}
\hline & Total EI & $\begin{array}{c}\text { Perception } \\
\text { of } \\
\text { Emotion }\end{array}$ & $\begin{array}{l}\text { Managing } \\
\text { Own } \\
\text { Emotions }\end{array}$ & $\begin{array}{l}\text { Managing } \\
\text { Others' } \\
\text { Emotions }\end{array}$ & $\begin{array}{l}\text { Utilization } \\
\text { of Emotion }\end{array}$ \\
\hline \multicolumn{6}{|l|}{ Adjusted $R^{2}$} \\
\hline M1. Extraversion & .44 & .28 & .46 & .27 & .03 \\
\hline M2. Item GFP & .39 & .28 & .52 & .15 & .00 \\
\hline M3. HEXACO Domains & .55 & .33 & .55 & .44 & .22 \\
\hline M4. HEXACO Facets & .65 & .41 & .64 & .52 & .32 \\
\hline \multicolumn{6}{|l|}{ Model Comparisons } \\
\hline \multicolumn{6}{|l|}{ M1 versus M3 } \\
\hline Adjusted $R^{2}$ Change & .11 & .05 & .09 & .17 & .20 \\
\hline $95 \% \mathrm{CI}$ & {$[.08, .13]$} & {$[.03, .08]$} & {$[.06, .11]$} & {$[.14, .20]$} & {$[.16, .24]$} \\
\hline \multicolumn{6}{|l|}{ M2 versus M3 } \\
\hline Adjusted $R^{2}$ Change & .16 & .05 & .02 & .29 & .22 \\
\hline $95 \%$ CI & {$[.13, .20]$} & {$[.02, .09]$} & {$[.00, .05]$} & {$[.25, .34]$} & {$[.18, .26]$} \\
\hline \multicolumn{6}{|l|}{ M3 versus M4 } \\
\hline Adjusted $R^{2}$ Change & .10 & .08 & .09 & .08 & .10 \\
\hline $95 \% \mathrm{CI}$ & {$[.08, .12]$} & {$[.06, .11]$} & {$[.07, .12]$} & {$[.05, .10]$} & {$[.07, .13]$} \\
\hline
\end{tabular}

Note. All models are statistically significant $(\mathrm{p}<.05)$. The four models had the following predictors: $\mathrm{M} 1=$ extraversion; M2 = Item GFP; M3 = 6 HEXACO domains; M4 $=25$ HEXACO facets. 
Table 5

Standardized Regression Coefficients for Regression Model Predicting Trait EI Factors from HEXACO Domains

\begin{tabular}{lccccc}
\hline \multicolumn{1}{c}{ Predictor } & $\begin{array}{c}\text { Total } \\
\text { EI }\end{array}$ & $\begin{array}{c}\text { Perception } \\
\text { of } \\
\text { Emotion }\end{array}$ & $\begin{array}{c}\text { Managing } \\
\text { Own } \\
\text { Emotions }\end{array}$ & $\begin{array}{c}\text { Managing } \\
\text { Others' } \\
\text { Emotions }\end{array}$ & $\begin{array}{c}\text { Utilization } \\
\text { of } \\
\text { Emotion }\end{array}$ \\
\hline Honesty-humility & .03 & .06 & .01 & -.01 & .02 \\
Emotionality & $.20^{*}$ & $.10^{*}$ & $-.10^{*}$ & $.41^{*}$ & $.34^{*}$ \\
Extraversion & $.59^{*}$ & $.46^{*}$ & $.52^{*}$ & $.54^{*}$ & $.21^{*}$ \\
Agreeableness & .03 & .02 & $.08^{*}$ & $.10^{*}$ & $-.11^{*}$ \\
Conscientiousness & $.16^{*}$ & $.15^{*}$ & $.22^{*}$ & .04 & .00 \\
Openness & $.24^{*}$ & $.15^{*}$ & $.13^{*}$ & $.18^{*}$ & $.31^{*}$ \\
\hline
\end{tabular}

$* p<.01$ 


\section{Item Level Analysis of Trait EI}

\section{Online Supplement}

Table S1 provides item-level descriptive statistics, loadings of trait EI items on the first unrotated factor, and correlations of Item GFP with the Trait EI items. Items measuring perception of emotions all loaded highly on the first unrotated factor. In general, items with the strongest loadings tended to reflect self-rated emotion-related abilities, optimism, and well-being. Utilization of emotions items had the smallest loadings on the first factor. In general, items that loaded more weakly on the first factor reflected styles, approaches, and preferences along with beliefs about the importance of emotions in life rather than abilities. Altogether, the correlation of an item with the first factor broadly mirrored the pattern of correlations with the GFP, but this was far from perfect. Item agreement also mirrored these loadings and GFP correlations, whereby items with low-levels of agreement loaded negatively and items with high-levels of agreement loaded highly. 
Table S1

Descriptive Statistics, Loadings on First Factor Component, and Correlations with the General Factor of Personality for Trait Emotional Intelligence Items

\begin{tabular}{|c|c|c|c|c|c|}
\hline & & & & & GFP \\
\hline Text & $\mathrm{M}$ & SD & $\% \mathrm{~A}$ & EI r & $\mathrm{r}$ \\
\hline 25. PER: I am aware of the non-verbal messages other people send. & 4.02 & 0.76 & 84 & .71 & .38 \\
\hline 18. PER: I tend to misread peoples' facial expressions. & 2.07 & 0.76 & 6 & -.67 & -.42 \\
\hline 5. PER: I find it hard to understand the non-verbal messages of other people. & 2.15 & 0.95 & 11 & -.66 & -.38 \\
\hline 15. PER: I am aware of the non-verbal message that I send others. & 3.73 & 0.85 & 72 & .63 & .35 \\
\hline 22. PER: I easily recognize my emotions as I experience them. & 3.96 & 0.69 & 83 & .59 & .34 \\
\hline 32. PER: I find it hard to tell how someone is feeling from their tone of voice. & 2.14 & 0.79 & 8 & -.54 & -.33 \\
\hline 9. PER: I am aware of my emotions as I experience them. & 4.16 & 0.61 & 92 & .53 & .30 \\
\hline 33. PER: It is difficult for me to understand why people feel the way they do. & 2.21 & 0.80 & 8 & -.52 & -.34 \\
\hline 4. OTH: Other people find it easy to confide in me. & 4.14 & 0.71 & 85 & .47 & .34 \\
\hline 12. OWN: When I experience a positive emotion, I know how to make it last. & 3.55 & 0.78 & 57 & .47 & .41 \\
\hline 29. PER: I know what other people are feeling just by looking at them. & 3.31 & 0.86 & 47 & .47 & .11 \\
\hline 10. OWN: I generally don't expect good things to happen. & 2.27 & 0.99 & 13 & -.45 & -.53 \\
\hline 23. OWN: I motivate myself by imagining a good outcome to tasks I take on. & 4.00 & 0.70 & 83 & .45 & .40 \\
\hline 19. PER: I often don't know why my emotions change. & 2.39 & 0.94 & 16 & -.45 & -.48 \\
\hline 1. OTH: I know when to speak about my personal problems to others. & 3.91 & 0.82 & 80 & .44 & .31 \\
\hline $\begin{array}{l}\text { 2. OWN: When I am faced with obstacles, I remember times when I faced similar } \\
\text { obstacles and overcame them. }\end{array}$ & 4.08 & 0.75 & 86 & .44 & .40 \\
\hline 24. OTH: I compliment others when they have done something well. & 4.49 & 0.56 & 98 & .43 & .35 \\
\hline 30. OTH: I help other people feel better when they are down. & 4.08 & 0.61 & 90 & .42 & .22 \\
\hline 28. OWN: When I am faced with a challenge, I give up because I believe I will fail. & 1.72 & 0.73 & 3 & -.42 & -.62 \\
\hline 14. OWN: I seek out activities that make me happy. & 4.03 & 0.63 & 87 & .38 & .19 \\
\hline 13. OTH: I arrange events others enjoy. & 3.78 & 0.82 & 71 & .37 & .29 \\
\hline 3. OWN: I generally expect to fail when I try something new. & 2.05 & 0.87 & 8 & -.37 & -.44 \\
\hline 21. OWN: I find it hard to control my emotions. & 2.27 & 0.91 & 12 & -.36 & -.58 \\
\hline 31. OWN: I use good moods to help myself keep trying in the face of obstacles. & 3.64 & 0.82 & 64 & .35 & .12 \\
\hline 17. UTI: When I am in a positive mood, solving problems is easy for me. & 4.10 & 0.67 & 86 & .32 & .14 \\
\hline 20. UTI: I don't find that being in a positive mood helps me come up with new ideas. & 2.35 & 0.87 & 12 & -.29 & -.21 \\
\hline $\begin{array}{l}\text { 6. UTI: Some of the major events of my life have led me to re-evaluate what is } \\
\text { important and not important. }\end{array}$ & 4.33 & 0.68 & 92 & .28 & .19 \\
\hline $\begin{array}{l}\text { 26. OTH: When another person tells me about an important event in his or her life, I } \\
\text { almost feel as though I have experienced the event myself. }\end{array}$ & 3.11 & 0.90 & 37 & .25 & .05 \\
\hline 27. UTI: When I feel a change in emotions, I tend to come up with new ideas. & 3.23 & 0.75 & 35 & .24 & .01 \\
\hline 16. OTH: I have little interest in the impression I make on others. & 2.35 & 0.85 & 12 & -.23 & -.11 \\
\hline 11. OTH: I prefer to keep my emotions private. & 3.41 & 0.97 & 55 & -.14 & -.01 \\
\hline 8. UTI: Emotions don't have much effect on my quality of life. & 2.30 & 0.92 & 14 & -.11 & .10 \\
\hline 7. UTI: When my mood changes I see new possibilities. & 3.40 & 0.84 & 49 & .08 & -.18 \\
\hline
\end{tabular}

Note. \% A is percentage agreeing or strongly agreeing with statement. EI $r$ is the loading on the first unrotated factor of all items. GFP $r$ is the correlation with the item-level GFP. PER = Perception of emotion, OWN = Managing own emotions, OTH = Managing others' emotions, $\mathrm{UTI}=$ Utilization of emotion. 
Facet Level Regression Model

Table S2 provides facet-level regression coefficients for models predicting Trait EI. Variance explained is presented in the primary manuscript.

Table S2 Regression Coefficients for Models Predicting Trait EI from HEXACO Facets

\begin{tabular}{|c|c|c|c|c|c|c|c|c|c|c|c|c|c|c|c|}
\hline \multirow[b]{2}{*}{ Predictor } & \multicolumn{3}{|c|}{ Total EI } & \multicolumn{3}{|c|}{$\begin{array}{l}\text { Perception of } \\
\text { Emotions }\end{array}$} & \multicolumn{3}{|c|}{$\begin{array}{l}\text { Managing Own } \\
\text { Emotions }\end{array}$} & \multicolumn{3}{|c|}{$\begin{array}{l}\text { Managing Others' } \\
\text { Emotions } \\
\end{array}$} & \multicolumn{3}{|c|}{$\begin{array}{c}\text { Utilization of } \\
\text { Emotions }\end{array}$} \\
\hline & Beta & $\mathrm{b}$ & se & Beta & $\mathrm{b}$ & se & Beta & $\mathrm{b}$ & se & Beta & $\mathrm{b}$ & se & Beta & $\mathrm{b}$ & se \\
\hline (Intercept) & .00 & .85 & .11 & .00 & .47 & .21 & .00 & .97 & .15 & .00 & .85 & .14 & .00 & 1.33 & .20 \\
\hline H1: Sincerity & -.02 & -.01 & .01 & .02 & .02 & .02 & -.04 & -.04 & .02 & -.05 & -.03 & .02 & -.02 & -.02 & .02 \\
\hline H2: Fairness & -.04 & -.02 & .01 & -.06 & -.05 & .02 & -.03 & -.02 & .02 & -.01 & -.01 & .02 & .02 & .01 & .02 \\
\hline H3: Greed-Avoidance & -.09 & -.05 & .01 & -.10 & -.07 & .02 & -.06 & -.04 & .01 & -.05 & -.03 & .01 & -.06 & -.04 & .02 \\
\hline H4: Modesty & .06 & .04 & .01 & .11 & .10 & .03 & .01 & .01 & .02 & .00 & .00 & .02 & .01 & .01 & .02 \\
\hline E1: Fearfulness & -.02 & -.01 & .01 & .00 & .00 & .02 & -.01 & -.01 & .02 & -.02 & -.01 & .01 & -.03 & -.02 & .02 \\
\hline E2: Anxiety & -.05 & -.02 & .01 & -.06 & -.05 & .02 & -.14 & -.09 & .02 & .01 & .01 & .02 & .12 & .08 & .02 \\
\hline E3: Dependence & .04 & .02 & .01 & -.06 & -.05 & .02 & -.01 & -.01 & .02 & .13 & .08 & .02 & .16 & .12 & .02 \\
\hline E4: Sentimentality & .26 & .16 & .01 & .26 & .25 & .03 & .09 & .08 & .02 & .26 & .18 & .02 & .15 & .12 & .03 \\
\hline X1: Social Self-Esteem & .26 & .16 & .02 & .23 & .21 & .03 & .27 & .21 & .02 & .11 & .08 & .02 & .12 & .10 & .03 \\
\hline X2: Social Boldness & .13 & .07 & .01 & .15 & .12 & .02 & .01 & .01 & .02 & .14 & .08 & .02 & .09 & .06 & .02 \\
\hline X3: Sociability & .04 & .02 & .01 & .05 & .04 & .02 & -.02 & -.02 & .02 & .17 & .10 & .02 & -.09 & -.06 & .02 \\
\hline X4: Liveliness & .17 & .10 & .02 & .05 & .05 & .03 & .30 & .22 & .02 & .10 & .06 & .02 & .04 & .03 & .03 \\
\hline A1: Forgiveness & .00 & .00 & .01 & -.01 & -.01 & .02 & -.01 & -.01 & .01 & .06 & .03 & .01 & -.05 & -.03 & .02 \\
\hline A2: Gentleness & -.06 & -.04 & .01 & -.12 & -.11 & .03 & .00 & .00 & .02 & -.02 & -.02 & .02 & -.01 & -.01 & .03 \\
\hline A3: Flexibility & -.01 & -.01 & .01 & .03 & .03 & .03 & -.01 & -.01 & .02 & -.04 & -.03 & .02 & -.04 & -.03 & .03 \\
\hline A4: Patience & .03 & .02 & .01 & .05 & .04 & .03 & .03 & .03 & .02 & .02 & .02 & .02 & -.04 & -.03 & .03 \\
\hline C1: Organization & .05 & .02 & .01 & .04 & .03 & .02 & .02 & .01 & .01 & .04 & .02 & .01 & .05 & .03 & .02 \\
\hline $\mathrm{C} 2$ : Diligence & .08 & .05 & .01 & -.01 & -.01 & .03 & .18 & .15 & .02 & .02 & .01 & .02 & .07 & .06 & .03 \\
\hline C3: Perfectionism & .04 & .02 & .01 & .05 & .05 & .02 & .00 & .00 & .02 & .04 & .02 & .02 & .02 & .02 & .02 \\
\hline C4: Prudence & .02 & .01 & .02 & .09 & .09 & .03 & .07 & .06 & .02 & -.02 & -.01 & .02 & -.18 & -.15 & .03 \\
\hline O1: Aesthetic Appreciation & .04 & .02 & .01 & .02 & .02 & .02 & .02 & .01 & .01 & .07 & .04 & .01 & .03 & .02 & .02 \\
\hline $\mathrm{O} 2$ : Inquisitiveness & -.05 & -.03 & .01 & -.04 & -.03 & .02 & -.01 & -.01 & .02 & -.07 & -.04 & .02 & -.04 & -.03 & .02 \\
\hline O3: Creativity & .17 & .09 & .01 & .12 & .10 & .02 & .11 & .08 & .02 & .12 & .08 & .02 & .17 & .13 & .02 \\
\hline O4: Unconventionality & .08 & .05 & .01 & .05 & .05 & .03 & .04 & .03 & .02 & .01 & .01 & .02 & .15 & .13 & .03 \\
\hline I: Altruism & .16 & .12 & .02 & .07 & .08 & .03 & .09 & .08 & .02 & .21 & .17 & .02 & .18 & .17 & .03 \\
\hline
\end{tabular}

Note. Absolute standardized betas above .10 are in bold. 